\title{
Influence of Light Polarization on Photoswitching of Fulgimide Monolayers on surfaces
}

\author{
Stefan Klaes ${ }^{\mathrm{a}}$, Catherine Henry de Villeneuve ${ }^{\mathrm{a} *}$, François Ozanam ${ }^{\mathrm{a}}$, \\ Christoph Barta ${ }^{\mathrm{b}}$, Karola Rück-Braun ${ }^{\mathrm{b}}$ Rémi Métivier $^{\mathrm{c}}$ and Philippe Allongue ${ }^{\mathrm{a} *}$ \\ a) Laboratoire de Physique de la Matière Condensée, Ecole Polytechnique, CNRS, IP Paris, \\ 91128 Palaiseau, F (France) \\ b) Institut für Chemie, Technische Universität Berlin, Strasse des 17 Juni 135, \\ D-10623 Berlin (Germany) \\ c) PPSM, ENS Cachan, CNRS, Université Paris-Saclay, 94235 Cachan (France)
}

\begin{abstract}
:
The photo isomerization characteristic parameters (photo-stationary states (PSS), cross section $\sigma$, quantum yield $\eta$ ) of indolyl fulgimide compounds dispersed in solution are quantitatively compared to those of the same compounds covalently immobilized as a monolayer on a functionalized Si(111) surfaces (see Advanced Materials 25, 416 (2013)). The photo isomerization kinetics of fulgimide compounds in solution is monitored using UV-Vis spectroscopy and that of monolayers by in situ calibrated FTIR spectroscopy. While the isomeric composition at photo stationary states are very similar in solution and at surfaces, a strong influence of the incident light polarization is found on photo isomerization cross section $\sigma\left(\sigma_{s} \ll \sigma_{p}<\sigma_{s o l}\right)$. Accounting for the local excitation electromagnetic field indicates that the fulgimide groups adopt a preferential tilt angle with respect to the surface normal and that their transition dipole is almost parallel to the surface plane for either $\mathrm{C}$ or $\mathrm{E} / \mathrm{Z}$ isomers. DFT indicates that this orientation corresponds to photochromic groups that are lying in contact with the underlying monolayer. This interaction plausibly explains why the quantum yield of photoisomerization is found nearly twice lower at the surface than in the solution.
\end{abstract}

* Corresponding authors: Catherine Henry de Villeneuve (e-mail: catherine.henry-devilleneuve@polytechnique.edu); Philippe Allongue (e-mail: philippe.allongue@ polytechnique.edu);

Keywords: Fulgimide, Photochromism, surface functionalization, monolayer, silicon 


\section{Introduction:}

Photochromic molecules are molecules capable of changing conformation upon absorption of electromagnetic radiation [1]. This unique property is exploited in many different fields. For instance photochromic molecules were incorporated into polymers [2] [3] or thin films [4] [5] [6] [7] [8] [9] [10] to realize multifunctional layers, in particular to store information [11] [12] [13] or modulate charge transport [14] [15]. Photochromic molecules can also be used in the context of biological systems [16], to structure soft matter with light [10], to control the function of biomaterials [17], or to promote catalytic activity [18]. Immobilization of photochromic molecules on surfaces [19] [20] [21] [22] [23] [24] [25] [26] [27], very often on gold, or self-assembled as Langmuir-Blodgett (LB) layers [28] is a further approach to control wettability of surfaces with external light [29], which is crucial in microfluidics, or to tailor surface conductance [30] [31] [32] [33] [34] and surface work function.[35] [36] [37] [38] Photochromic molecules can be also adsorbed on nanoparticles to control their properties [39].

On the molecular scale, photo isomerization implies the absorption of a photon by the molecule which promotes the molecule to a Frank-Condon state before the excited electron relaxes to a lower-energy state which brings the photochromic molecules either to its starting state or to another isomeric state [40]. The probability of the latter event corresponds to the quantum yield $(\eta)$ and it has been established that $\eta$ depends on chemical substituents and polarity of the solvent in the case of azobenzenes [41] [42] [43] [44], fulgides [45] [46] [47,48] and spiropyrans [49] [50] [51] or diarylethenes [52] [53] dispersed in a solvent. The behaviour at the surface depends strongly on the architecture of the assembly (for a review at metallic surfaces see Ref. [40]). When the photoswitching molecules are in direct contact with the substrate, the photoswitching occurs but seems very slow $\{$ Comstock, $2007 \# 758\}\{$ Safiei, 2012 \#752\}. This may partly due to steric hindrance, chemical and/or electronic coupling with 
the substrate. This motivated studies aiming at increasing the free volume around photochromic compounds [56] [19] [57,58] and to reduce the electronic coupling with the substrate [59] [33] [31] using chemical spacers or a semimetal as substrate \{Nickel, 2017 $\# 1352\}$. In such constructs the photo kinetics of photo isomerization of photochromic molecules, characterized by its effective cross section $(\sigma)$, may be as fast as in solution. This has been demonstrated with azobenzene derivatives \{Wagner, $2009 \# 1473$ \} \{Krekiehn, 2015 $\# 1286$ \} $\{$ Moldt, 2016\#1409\}. However $\sigma$ is yet found to be much smaller than in solution for fulgimides tethered on an organic monolayer on silicon\{Schulze, $2015 \# 1314$ \} or spirosyran \{Nickel, $2017 \# 1352$ \} evaporated on $\mathrm{Bi}(111)$, which minimizes electronic coupling. steric effects, mechaniealand/or electronic compling with the substrate are frequently involed to explain the different behaviour at sufaces. and this motivated various strategies to inerease

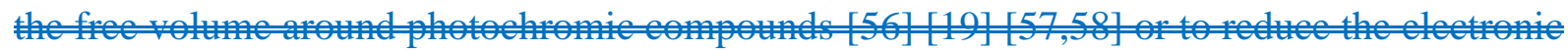
eopling wh sth [59][33][31] small density of states at Fermi level [26]. In spite of sionifieant improments, when the ph than the value measured for the same photochromic molecule dispersed in a solvent and the behaviour at the sufface depends on the arehitecture of the assembly (for a review at metllie sufaces

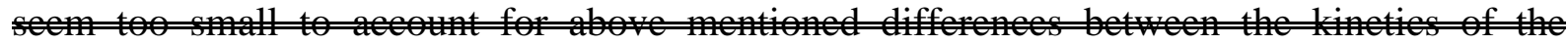
One key physical effect that has been largely ignored in the past is the influence of the local excitation electromagnetic field $\vec{E}_{\text {loc }}$ although it is well known that photochemistry is strongly influenced by the presence of a substrate [62]. Such a knowledge appears however indispensable to discuss the influence of local chemical environment on the photoisomerization cross sections. 
In this work we have investigated the photoswitching properties of fulgimide compounds because they present the key advantage of a very good thermal stability and a large quantum yield [45]. Scheme 1 displays the structure of the fulgimide isomers and solid arrows indicate optical transitions under visible $(\lambda \sim 525 \mathrm{~nm})$ and UV light $(\lambda \sim 365 \mathrm{~nm})$. Both wavelengths refer to the main absorption band of the respective isomers. Visible irradiation promotes ring opening which transforms the closed form $(\mathrm{C})$ into the open form (E). UV irradiation leads to the back formation of the C-isomer but also of another open form (Z-isomer). Amino-fulgimide may be immobilized as a monolayer on a surface (see scheme 2) [22]. The photo isomerization kinetics are monitored in solution using UV-Vis spectroscopy and at surfaces using in situ calibrated FTIR spectroscopy. A new experimental protocol has been developed with respect to Ref. [22] in order to determine the photo isomerization cross section $(\sigma)$ and the composition of the surface at the photo-stationary states (PSS) with high precision. In the case of fulgimide MLs, $\sigma$ is investigated as a function of the incident light polarization and the fulgimide surface density. Results are discussed within the framework of a molecular model and the properties of the local electromagnetic field.

\section{Experimental:}

All chemicals used for surface modification (purchased from Aldrich) were of the highest available purity and used as received. Indolyl fulgimide compounds substituted with an ethylamino linker were synthesized as detailed in reference [21]. For silicon etching, electronicgrade chemicals (Carlo Erba, RSE) were used. ATR-FTIR silicon prisms were cut from double-side polished (FZ-purified, $800 \Omega . \mathrm{cm}$ ) n-type $\operatorname{Si}(111)$ wafers with $0.2^{\circ}$ miscut angle along the $[11 \overline{2}]$ direction (Sil'tronix, France). For AFM observations one-side polished (CZ purified, 1-10 $\Omega . c m$, with a similar miscut) were used. The silicon (111) surface was first treated by anisotropic etching in oxygen-free $40 \% \mathrm{NH}_{4} \mathrm{~F}$ solution as reported before to obtain 
clean H-terminated $\operatorname{Si}(111)$ surfaces with wide and atomically flat terraces separated by bilayer-high steps $(3.1 \AA)$. [63,64]

Monolayer characterizations: FTIR characterizations were performed in ATR geometry with an Equinox 55 spectrometer (Bruker, Germany) equipped with a liquid-nitrogen cooled Mercury-Cadmium Telluride photovoltaic detector. The ATR samples (thickness 500-550 $\mu \mathrm{m})$ were shaped as prisms with two opposite sides bevelled at $\sim 45^{\circ}$. Their length $(\sim 15 \mathrm{~mm}$, for ca 30 internal reflections) was chosen to obtain wide range FTIR spectra (1000- $4000 \mathrm{~cm}^{-}$ ${ }^{1}$ ). Measurements were carried out in a $\mathrm{N}_{2}$-purged ATR chamber to minimize the contribution of $\mathrm{H}_{2} \mathrm{O}$ vapour and $\mathrm{CO}_{2}$ absorption bands. Spectra were recorded in s- and p-polarization.

\section{In situ monitoring of photoswitching:}

In the case of monolayers, photo switching was monitored by in situ FTIR spectroscopy using a specifically home-designed optical experimental setup installed within the custom chamber of the IR spectrophotometer (see scheme in supplementary information, section S1). Both sides of the sample were irradiated with a quasi-parallel beam using LEDs (UV: $365 \mathrm{~nm}, 35.9$ $\mathrm{mW} / \mathrm{cm}^{2}$; Vis: $525 \mathrm{~nm}, 51.5 \mathrm{~mW} / \mathrm{cm}^{2}$ ) and cylindrical lenses. Neutral filters were used to adjust the photon flux. For most of the experiments, a $45^{\circ}$ incidence angle is used. Polarizers were added for experiments with p-polarized light. For experiments with s-polarized light, it has been found more reliable to work at normal incidence rather than using polarizers, in order to ensure the absence of any residual electromagnetic-field component normal to the surface. A computer controlled shutter allowed for irradiating the sample with short light pulses (few ms) at the very initial stages of the photo-switching and longer ones (1-100 s) at the subsequent stages to control the photon dose. Photon fluxes were calibrated in each configuration with a photodiode to determine the photon dose during light pulses. The 
nominal photon flux (in the absence of neutral filters and polarizers) was $6.610^{16}$ photons $\mathrm{cm}^{-}$ ${ }^{2} \mathrm{~s}^{-1}$ and $1.410^{17}$ photons $\mathrm{cm}^{-2} \mathrm{~s}^{-1}$ for UV and visible light, respectively. FTIR spectra (150 scans, resolution $4 \mathrm{~cm}^{-1}$ ) were recorded between light pulses, i.e., in the dark where the system is stable. This procedure allows for recording FTIR spectra corresponding to controlled photon dose for an accurate determination of the photokinetics.

Photo switching in solution was characterized with a similar approach using in situ UV-Vis spectroscopy. $20 \mu \mathrm{M}$ of either E- or C-fulgimide were dissolved in acetonitrile. A simplified optical setup was installed in the spectrophotometer (Agilent Technologies, Cary 50) to monitor the process. The nominal photon flux (in the absence of neutral filters and polarizers) was $3.310^{16}$ photons $\mathrm{cm}^{-2} \mathrm{~s}^{-1}$ and $2.210^{17}$ photons $\mathrm{cm}^{-2} \mathrm{~s}^{-1}$ for UV and visible light, respectively. A similar protocol, recording UV-Vis spectra between light pulses, was used.

Preparation of fulgimide monolayers with variable surface densities: Pure C- and Efulgimide were synthesized as reported in ref. [21] and immobilized on the silicon surface using the multistep protocol described in Scheme 2. The primer $\mathrm{COOH}$-terminated monolayer (step 1) was grafted by photochemical reaction (wavelength $312 \mathrm{~nm}$ ) between the $\mathrm{H}$ terminated surface and neat undecylenic acid as detailed in Ref. [65]. The "activation" of the carboxylic groups (step 2) was performed in MES buffer ( $\mathrm{pH}=5,15^{\circ} \mathrm{C}$, under Ar bubbling) containing N-Hydroxysuccinimide (HOSuc, 100 mM and 1-ethyl-3-(3-dimethylaminopropyl)carbodiimide (EDC, $100 \mathrm{mM})$. The reaction time was $1 \mathrm{~h}$. The samples were then rinsed successively $\left(10 \mathrm{~min}, 40^{\circ} \mathrm{C}, \mathrm{pH}=5\right)$ in $0.1 \mathrm{M} \mathrm{NaH}_{2} \mathrm{PO}_{4}, 0.01 \mathrm{M} \mathrm{NaH} \mathrm{PO}_{4}$, in ultra-pure water and blown dry using an Ar stream. The coupling of the fulgimide (step 3) was conducted in an acetonitrile solution containing $5 \mathrm{mM}$ of fulgimide ( $2 \mathrm{~h}$ at room temperature, in the dark). More details are given in Ref. [22]. To vary the photochromic molecule density, step 3 
(scheme 2) was also conducted in an acetonitrile solution containing amino-fulgimide and ethylamine. An excess of fulgimide was used to account for the slower coupling rate of fulgimides (4000 $\mathrm{s}^{-1}$ ) with respect to that of ethylamine (200 $\mathrm{s}^{-1}$ ) (measured from complementary in situ FTIR measurements, data not shown). Ethylamine /fulgimide ratios of $0.17,0.26,0.33$ and 0.66 were used.

Full range FTIR spectra recorded at the different steps of the preparation of the fulgimide MLs are given in supplementary information (section S2). They are perfectly consistent with previous results [22]. Also explained in supplementary information (section S2) all samples studied in this work present a contamination level that is below 0.1 equivalent monolayer. The chemical composition of all surfaces was quantitatively determined from careful calibration of the $\mathrm{vC}=\mathrm{O}$ band at $1715 \mathrm{~cm}^{-1}$ related to $\mathrm{COOH}$ groups [65] [66]. More details are given in supplementary information, section S2. e

\section{Computational methods:}

The geometries of the fulgimide compound in the $\mathrm{C}, \mathrm{E}$ and $\mathrm{Z}$ forms were fully optimized using the Becke-3-Lee-Yang-Parr (B3LYP) exchange functional [67] with the 6-311+G(d,p) basis set, in vacuum, as implemented in the Gaussian 09 software package [68]. The absence of negative frequencies was checked to ensure true minima for all geometries. Frank-Condon energy transitions and corresponding transition dipole moments were calculated using the time-dependent DFT formalism (TD-DFT) with the same functional and basis set. As explained in the text, the functionalized monolayer on $\operatorname{Si}(111)$ surface was sketched without minimization. The DFT cell consists of alkylamide molecules as spacer chains (optimized separately) along a $3.84 \times 6.65 \AA$ rectangular $2 \mathrm{D}$-assembly and one fulgimide-terminated chain. 


\section{Results:}

\subsection{Photoswitching in solution vs. at surfaces:}

Figure 1a shows series of UV-Vis spectra of a $20 \mu \mathrm{M}$ C-solution (in acetonitrile) irradiated with $525 \mathrm{~nm}$ light at nominal photon flux. The red line is the initial spectrum (C-form) and the blue line is the last spectrum of the experiment. The main absorption band around $530 \mathrm{~nm}$ (red line), and that around $350 \mathrm{~nm}$ (blue line) are in perfect agreement with those reported in the literature for the C- and E-isomers, respectively [47]. All intermediate spectra (grey lines) can be accounted for by a linear combination of the spectrum of the initial pure C-solution and that of the final solution. The relative weight of the coefficients in this combination provides the relative advancement $0<\alpha<1$ of the photo isomerization reaction, as plotted in panel (b), (black squares, left y-scale), for the first Vis illumination. Keeping the pure Csolution as the reference state, the advancement of the backward photoisomerization under UV is also plotted in panel (b) (open squares). The composition along the photoconversion was simply determined by fitting each intermediate spectrum with a linear combination of the spectra of solutions composed of pure E- and C-isomers of known concentrations (full line, right scale in panel b).

Figure 1c displays narrow range FTIR spectra recorded during the photo switching of an initially pure and dense C-monolayer. The surface density of immobilized fulgimide was determined as explained in section S2 of the supplementary information. It is $1.6 \mathrm{C}$ isomers $/ \mathrm{nm}^{2}$ for this sample. The initial spectrum (red line) presents two main IR bands at $1698 \mathrm{~cm}^{-1}$ and $1758 \mathrm{~cm}^{-1}$ assigned to asymmetric and symmetric $\mathrm{vC}=\mathrm{O}$ stretching modes of maleimide groups. Upon visible illumination $(525 \mathrm{~nm})$ these two bands progressively shift towards high frequency and their intensity varies. At the end of the experiment (blue line), the positions of the two bands are $1689 \mathrm{~cm}^{-1}$ and $1738 \mathrm{~cm}^{-1}$, which is very close to the positions measured for a pure E-surface $\left(1690 \mathrm{~cm}^{-1}\right.$ and $\left.1739 \mathrm{~cm}^{-1}\right)$. All the above observations are in 
perfect accordance with previous work [21] [22]. The advancement $\alpha$ of the photoisomerization reaction was determined from differential spectra (see inset of panel c), taking as the reference spectrum that of the initial C-terminated surface: the blue solid line in the inset represents the difference between the blue and red lines shown in the main frame of panel (c). Within this representation, all the intermediate spectra (grey lines) are precisely proportional to the final difference, which straightforwardly provides $\alpha$ for the first Vis irradiation (black squares in panels (d)). Open squares correspond to $\alpha$ during the subsequent UV irradiation period, keeping the initial $\mathrm{C}$-surface as the reference surface. To be quantitative, $\alpha$ was transformed into a number of switched isomers, which is given by the right $\mathrm{y}$-scale in panels (b) and (d). It was done using a specific calibration of the vCO bands of the $\mathrm{C}$ and $\mathrm{E}$ isomers.

Comparison of panels (b) and (d) in Fig. 1 immediately points out that in both cases a large amount $\mathrm{C}$-isomers have switched under visible irradiation but that the subsequent UV illumination does not fully restores all the initial C-forms (Fig. 1b, symbols). In solution, the first isomerization under Vis is completed within ca 10 s with $100 \%$ of the $\mathrm{C}$ isomers transformed into E forms. The subsequent UV illumination restores $77 \%$ of the initial $\mathrm{C}$ isomers. At the surface, using a similar photon flux, it takes $100 \mathrm{~s}$ under Vis illumination to reach a quasi-steady-state corresponding to the switching of only $87 \%$ of the initial Cisomers. The back isomerization under UV light is also slower and less efficient -in terms of back conversion yield- since it restores only $61 \%$ of the switched C-isomers. Reaching PSS under UV is possible but requires longer exposure times [22]. This also leads to some photodegradation of the monolayer. In the following we only present experiments corresponding to short illumination times, leading to incomplete photoconversion.

The data in Fig. 1 may be replotted as a function of the photon dose $p$, as justified in supplementary information (Fig. S5) to determine the photo isomerization cross section $(\sigma)$ 
and the isomeric compositions at the photo-stationary state (PSS) as it is explained in the Appendix. It is found that $\sigma$ is approximately constant in the initial stages of the photochromic reaction but then decays above a critical photon dose $p^{*}$ (see Fig. A1). In other words only a fraction of the monolayer reacts upon illumination according to a single exponential law with a well-defined value of the cross section $\sigma$. In the following we only discuss this value of $\sigma$. The decay of $\sigma$ above $p^{*}$ will be the subject of a separate article. Notice that the same analysis gives a single exponential law for the complete photoisomerization of C-isomers dissolved in acetonitrile (under Vis light). Under UV, $\sigma$ decays (by 20\%) for high-enough photon doses.

\subsection{Isomeric composition at PSS}

Panel (a) of figure 2 presents the evolution of the isomeric composition upon consecutive UV/Vis illumination cycles, starting from a dense E-surface $\left(1.8\right.$ E-isomers $\left./ \mathrm{nm}^{2}\right)$. About 50\% of the monolayer is switched under each light exposure. Panel (b) of Fig. 2 gives the surface composition at $\mathrm{PSS}_{\mathrm{UV}}$ (left bar) and $\mathrm{PSS}_{\mathrm{Vis}}$ (right bar) for each cycle, determined as explained in the Appendix. The composition is given as $(\mathrm{C})$ and $(\mathrm{E} / \mathrm{Z})$ surface densities because $\mathrm{E}-$ and Z-forms cannot be discriminated by FTIR. The data analysis according to the procedure in the Appendix also yields the cross-section values ( $\sigma_{\mathrm{UV}}$ and $\left.\sigma_{\mathrm{Vis}}\right)$. Fig. S6 supports this analysis since the resulting $\sigma_{U V}$ and $\sigma_{\mathrm{Vis}}$ keep the same values over the successive illumination cycles.

Overall the isomeric compositions at PSS appear rather stable upon successive UV/Vis irradiation cycles. A slight enrichment of the surface in C-isomers is noticeable at PSS $_{\mathrm{UV}}$ after 6 irradiation cycles. The same trend is observed for $\mathrm{PSS}_{\mathrm{Vis}}$. The isomeric compositions at $\mathrm{PSS}_{\mathrm{Vis}}$ and $\mathrm{PSS}_{\mathrm{UV}}$ in solution and at surfaces with various densities of immobilized fulgimides are compared in Figure 3. In each case the left bar provides $\mathrm{PSS}_{\mathrm{Vis}}$ and the right one $\mathrm{PSS}_{\mathrm{UV}}$ after the first visible and the subsequent UV irradiation, starting from a pure C-solution or a 
pure C-monolayer. The isomeric compositions at PSSs are also summarized in Table 1. One may notice that the $\mathrm{PSS}_{\mathrm{UV}}$ composition in solution departs from $100 \% \mathrm{C}$. Indeed, the presence of a significant proportion of open forms at $\mathrm{PSS}_{\mathrm{UV}}$ confirms that an additional reaction $\mathrm{C} \rightarrow \mathrm{E}$ must be taken into account under UV in Scheme 1 since the C-isomer presents an absorption band around $350 \mathrm{~nm}$ in Fig. 1a. For a relevant comparison of the PSSs in solution and at surfaces we consider monolayers with less than $D^{*} \sim 0.9$ isomers $/ \mathrm{nm}^{2}$ because steric hindrance may be neglected. Steric hindrance is expected for denser monolayers [22]. With this precaution, Table 1 shows that the $\mathrm{PSS}_{\mathrm{Vis}}$ is equal in solution and at the surface and that the $\mathrm{PSS}_{\mathrm{UV}}$ is somewhat richer in E/Z isomers at the surface than in the solution.

\subsection{Photokinetics at surfaces}

The results of Figure 4 have been obtained on a sample with an initial isomer density of 1.6 E-isomers $/ \mathrm{nm}^{2}$. After a few incomplete photoswitching cycles like in Fig. 2, the starting surface consists of $\mathrm{C}$ and $(\mathrm{E}+\mathrm{Z})$ isomers. Therefore, $\ln \left(\frac{\left[C_{P S S}\right]-[C]}{\left[C_{P S S}\right]-\left[C_{\text {start }}\right]}\right)$ and $\ln \left(\frac{\left[E_{P S S}\right]-[E]}{\left[E_{P S S}\right]-\left[E_{\text {start }}\right]}\right)$ have been plotted as a function of the photon dose $p$. Here $[\mathrm{C}]$ and $[\mathrm{E}]$ designate the surface concentration of closed $\mathrm{C}$ and open $(\mathrm{E}+\mathrm{Z})$ isomers and the suffix refers to the starting surface and surface at PSS. Data analysis indicates that the total number of isomers is constant over successive illumination cycles. As justified in the Appendix, data (symbols in Fig. 4) may be fitted to a single exponential kinetics (straight dashed lines) up to a critical photon dose $p^{*}$ (indicated by the vertical bars in the figures). The photo isomerization cross section $\sigma$ is the inverse value of the straight-line slope. Above $p^{*}$, whose value depends on photon energy and light polarization, the process no longer follows a simple exponential law (slow decay of $\sigma$ as in Fig. A1b,d).

For a given monolayer, the cross section $\sigma$ was measured for s- and p-polarization of the excitation light (red and blue open squares respectively) and compared to that obtained 
with non-polarized light (black open squares). The different $\sigma$ values are reported in the left three blocks of Table 2. The right block of Table 2 gives also the $\sigma$-values measured for isomers dispersed in acetonitrile. In solution, the absorption cross section can conveniently be measured and the isomerization quantum yield $\eta$ is simply determined as the ratio of $\sigma$ to $\sigma_{a b s}$. According to Table 2 the magnitude of $\sigma$ follows the trend s-pol (surface) $<$ p-pol (surface) $<$ solution for a given wavelength and $\sigma_{U V}>\sigma_{V i s}$ in all conditions. Complementary experiments (see Table S1) indicate that $\sigma$ is quasi-independent of the isomer surface density, suggesting that this parameter is well representative of the photo isomerization of the fraction of photochromic molecules that switch from one configuration to the other one without any specific (e.g., steric) limitation. The $\sigma$-values measured in solution are close to those reported previously $\left(\sigma_{\mathrm{UV}}=510^{-18} \mathrm{~cm}^{2}\right.$ and $\sigma_{\mathrm{Vis}}=210^{-18} \mathrm{~cm}^{2}$ [70]). The differences may come from the substituent and/or the solvent which are different in our work. Concerning the cross sections measured at the surface, our values with non-polarized light are also in fair agreement, within a factor $2-4$, with recently published data $\left(\sigma_{U V}=2.310^{-18} / \mathrm{cm}^{2}\right.$ and $\sigma_{\mathrm{Vis}} 1.2$ $10^{-18} / \mathrm{cm}^{2}$ ) for fulgimides immobilized on a silicon surface [71].

\section{Discussion}

All in all, the data in Table 2 are consistent with past literature and confirm that the experimental values of $\sigma_{\mathrm{UV}}$ or $\sigma_{\mathrm{Vis}}$ are smaller at the surface than in solution. To the best of our knowledge, the light polarization dependence of the photoswitching efficiency is quantitatively investigated for the first time. The main purpose of this section is therefore to discuss the photo isomerization cross section $\sigma$ and particularly its dependence on light polarization at surfaces. The quantum yield is also discussed.

As recalled in the introduction, photo isomerization implies that absorption of a photon by the molecule brings it into an excited transition state before it relaxes either in its 
initial state or into another isomeric state with a probability $\eta$ (quantum yield). Therefore, the photo isomerization cross section of a molecule may be written:

$$
\sigma=\eta \sigma_{a b s}
$$

The absorbance cross section $\sigma_{\mathrm{abs}}$ is proportional to:

$$
J=\frac{1}{N} \int_{\Omega_{\min }}^{\Omega_{\max }}\left|\vec{E}_{\text {loc }} \cdot \vec{P}\right|^{2} d \Omega
$$

This integral outlines that the absorption of a photon by a photochromic molecule does depend on the alignment between the local electric field $\vec{E}_{l o c}$ and the transition dipole $\vec{P}$ of the molecule.

In solution $\vec{P}$ is randomly oriented, $\Omega_{\min }=0, \Omega_{\max }=4 \pi$ and $N=4 \pi$ and $E_{l o c}^{2}=\frac{1}{n}\left(\frac{n^{2}+2}{3}\right)^{2} E_{v a c}^{2}[72]$, with $E_{v a c}$ the electric field in vacuum and $n$ the refractive index of the solvent ( $n=1.34$ for acetonitrile). Therefore $J=1 / 3 P^{2} E_{l o c}{ }^{2}=1.27 P^{2} E_{v a c}{ }^{2}$. In the case of a monolayer of photochromic molecules immobilized on a substrate, one must take into account two points: (i) $\vec{E}_{l o c}$ is affected by the close proximity of the substrate and (ii) $\vec{P}$ may be constrained in certain directions of space. To the best of our knowledge, these considerations were not discussed at surfaces in literature although light polarization is known to induce reorientation of azobenzene in a polymer matrix $[28,73]$.

\subsection{Photo isomerization cross section at surfaces:}

Figure 5 defines the relative orientation of $\vec{E}_{l o c}$ and $\vec{P} . \vec{E}_{l o c}$ is set within the (y,z) plane at an angle $\alpha$ with respect to the surface normal (direction $z$ ) and the orientation of $\vec{P}$ is given by its angle $\beta$ with respect to surface normal and the azimuth angle $\gamma$ of its projection on (x,y) plane. Two extreme hypotheses are considered. 
Within the first one, the immobilized fulgimides adopt a random orientation with respect to surface normal. In this case, $0 \leq \beta \leq \pi / 2,0 \leq \gamma \leq 2 \pi, \Omega_{\min }=0, \Omega_{\max }=2 \pi$ and $N=2 \pi$ in Eq. (2). The integral $J$ therefore reduces to $J=1 / 3 P^{2}\left|E_{l o c}\right|^{2}$, independently of $\alpha$, i.e., the polarization of the illumination. In that case the ratio $\frac{\sigma_{S}}{\sigma_{p}}=\frac{\left|E_{s}\right|^{2}}{\left|E_{p}^{\|}\right|^{2}+\left|E_{p}^{\perp}\right|^{2}}$ and it depends only on the illumination energy. Under UV light, $\frac{\sigma_{s}}{\sigma_{p}}$ would be $\sim 0.12$ and under Vis light $\frac{\sigma_{s}}{\sigma_{p}}$ would be $\sim 0.04$. These values are sufficiently different from the experimental ones $(\sim 0.23 \pm 0.05$ for either UV or Vis illumination, see Table 2) to rule out this hypothesis.

Within the second hypothesis, the fulgimide are constrained by their environment and adopt a specific orientation. In this second case an angle $\beta$ in Fig. 5 may probably be defined but the angle $\gamma$ is likely to be random between 0 and $2 \pi$. Therefore, $J$ reduces to $\left[\frac{1}{2} \sin ^{2}(\beta) \sin ^{2}(\alpha)+\cos ^{2}(\beta) \cos ^{2}(\alpha)\right] P^{2} E_{l o c}{ }^{2}$, where $\alpha$ depends on light polarization and $\vec{E}_{l o c}$ is given by Eqs. (S1-S2) in the supplementary information [74]. In s-pol conditions, $\alpha=$ $\pi / 2$ and $J=\frac{1}{2} \sin ^{2}(\beta)\left|E_{S}\right|^{2}$ with $E_{S}$ given by (Eq. S1). At the incidence $\theta=45^{\circ}$ and with ppol light, $J=P^{2}\left(\frac{1}{2} \sin ^{2}(\beta)\left|E_{p}^{\|}\right|^{2}+\cos ^{2}(\beta)\left|E_{p}^{\perp}\right|^{2}\right)$, with $\vec{E}_{\mathrm{p}}^{\|}$and $\vec{E}_{\mathrm{p}}^{\perp}$ the components of $\vec{E}_{l o c}$ parallel and perpendicular to the surface, which expressions are given in section $\mathbf{S 5}$ in the supplementary information. For the numerical evaluation, the dielectric constant of silicon was taken from Ref. [75] at the relevant wavelength. At the silicon/air interface these equations yield $\left|E_{p}^{\|}\right|^{2} \ll\left|E_{p}^{\perp}\right|^{2}$. Assuming that the isomerization quantum yield $\eta$ is independent on light polarization, the ratio $\frac{\sigma_{s}}{\sigma_{p}}$ can be computed as a function of $\beta$ either to UV or Vis illumination. By comparing to the experimental ratio, one obtains the value of $\beta$ corresponding to the UV and Vis transition dipoles. For the UV excitation, $\beta$ is found to be $\sim 74^{\circ}$ and for the Vis excitation, $\beta \sim 64^{\circ}$. In either case, $\vec{P}$ appears close to the surface plane. 
DFT and TD-DFT calculations were performed with the Gaussian 09 package with the B3LYP functional and the $6-311+\mathrm{G}(\mathrm{d}, \mathrm{p})$ basis set in vacuum, and provided the minimized geometries, relative energies and Franck-Condon electronic transition energies for the three isomers C, E and Z (Fig. 6a). Given the orientation of $\vec{P}$ of the two relevant isomeric forms C and $\mathrm{E}$ as derived from TD-DFT computations, the experimental values of $\beta$ mean that the $\mathrm{C}$ and E-forms are similarly oriented and are almost lying parallel to the surface plane. This large tilt angle between the direction of the transition moment of the molecules and the surface normal may be accounted for by a rotation around the $\mathrm{C}-\mathrm{C}$ and $\mathrm{C}-\mathrm{N}$ bonds of the amide linkage to the monolayer. The configuration of the fulgimide is therefore different from that assumed in Ref. [22] where a nearly upright orientation was postulated because this corresponds to the equilibrium configuration of the isolated molecule (the fulgimide molecule coupled to the carboxylic acid).

According to Fig. 6, the experimental angle $\beta=74^{\circ}$ implies that the fulgimide groups must be almost parallel to the surface plane. To check the feasibility of this configuration, the grafted monolayer was modelled with alkylamide molecules (optimized separately) assembled along a $3.84 \times 6.65 \AA$ rectangular mesh (see atomic model in Fig. 7a). This corresponds to a density of 3.9 chains grafted per $\mathrm{nm}^{2}$ which is the maximum packing density on a $\mathrm{Si}(111)$ surface [69]. The DFT cell is limited to 3 x 3 chains and a photochromic molecule in its Cform is attached on one site located at the perimeter of the rectangular cell. It is tilted such as to bring its transition dipole moment at an angle $\beta=74^{\circ}$ with respect to the surface normal. The model shown in Figs. 7b-c corresponds to a situation where a vacancy is assumed to be present in the center of the DFT cell. In the side view (Fig. 7c), one clearly sees that the photochromic molecule is partially embedded inside the molecular carpet. Though these structures are not an optimized configuration of the supramolecular assembly, which is beyond the DFT computations performed here, such a tilted configuration is energetically 
affordable and a similar energy is found if the C-fulgimide is anchored on any other sites at the perimeter of the DFT cell. Similar energies are also found with a tilted E-fulgimide instead of a C-fulgimide. As to be expected, however, the energy barrier becomes too large for either a C- or a E-fulgimide without the vacancy at the center of the 3 x 3 cell. This shows that the vacancies in the grafted monolayer play a crucial role.

The model presented in Fig. 7 closely corresponds to real molecular surface concentration since the DFT cell is equivalent to $\sim 3$ chains $/ \mathrm{nm}^{2}$ and $\sim 1$ fulgimide $/ \mathrm{nm}^{2}$ whereas a dilute fulgimide monolayer is composed of 2.8 chains grafted per $\mathrm{nm}^{2}$ and $0.9 \mathrm{C}$ fulgimide $/ \mathrm{nm}^{2}$. Experimentally, there are enough vacancies $\left(1.1 / \mathrm{nm}^{2}\right)$ to accommodate all the immobilized photochromic molecules with a tilt compatible with experimental results. Given the influence of the light polarization on the cross section, we therefore postulate that the majority of immobilized fulgimides must adopt such a tilted configuration, without excluding that a small fraction of the photochromic molecules may adopt a non-tilted configuration.

A tilted molecular configuration was not to be expected and appears counter intuitive. Explain its origin would require dedicated supramolecular calculation. Nevertheless, based on above observations, one may suspect that this configuration comes from strong interactions between the fulgimide and the acid-terminated monolayer prior to the formation of the amide bond. In fact a large angle $\beta$ is already measured after immobilization since the cross sections $\sigma_{s, p}$ are constant from the very first photoswitching cycle for either pure C- or E-fulgimide monolayers. This tilted configuration may be maintained upon successive photoswitching because there is a small difference between the $\beta$ values for the UV and Vis transition. This strongly suggests that the photochromic reaction mainly involves the $\mathrm{C} \rightarrow \mathrm{E}$ and $\mathrm{E} \rightarrow \mathrm{C}$ photoisomerization, with a negligible contribution of the $\mathrm{Z}$ form because the direction of $\vec{P}$ for 
the $\mathrm{Z}$ form is significantly rotated, with regard to the $\mathrm{N}-\mathrm{C}$ bond of the alkylamide chain, see Fig. 6c).

\subsection{Isomerization quantum yield at surfaces:}

The quantitative determination of the optical absorption of a monolayer on an absorbing substrate like silicon is difficult and an absolute value of the isomerization quantum yield $\eta$ (Eq. 1) appears out of reach here. However, the determination of $J$ in different environments and that of the angle $\beta$ allows for estimating the ratio of the isomerization quantum yield in solution with that at the surface.

On the basis of Eq. (1) and the above expressions of $\sigma$, it comes (for experiments at $45^{\circ}$ incidence angle in p polarization and normal incidence in s polarization):

$$
\begin{aligned}
& \frac{\sigma_{p}}{\sigma_{s o l}}=3 n\left(\frac{3}{n^{2}+2}\right)^{2}\left(\frac{1}{2} \sin ^{2}(\beta)\left|\frac{\sqrt{2} \sqrt{\varepsilon_{S i}-1 / 2}}{\varepsilon_{S i} / \sqrt{2}+\sqrt{\varepsilon_{S i}-1 / 2}}\right|^{2}+\cos ^{2}(\beta)\left|\frac{\varepsilon_{S i}}{\varepsilon_{S i} / \sqrt{2}+\sqrt{\varepsilon_{S i}-1 / 2}}\right| 2\right) \frac{\eta_{\text {surf }}}{\eta_{\text {sol }}} \\
& \text { and: } \quad \frac{\sigma_{s}}{\sigma_{\text {sol }}}=6 n\left(\frac{3}{n^{2}+2}\right)^{2} \sin ^{2} \beta\left|\frac{1}{1+\sqrt{\varepsilon_{S i}}}\right|^{2} \frac{\eta_{\text {surf }}}{\eta_{\text {sol }}} .
\end{aligned}
$$

Numerical evaluation of these expressions using the above $\beta$ values allows a comparison with the experimental values given in Table 2. For the UV transition, the computed values are $\frac{\sigma_{p}}{\sigma_{\text {sol }}}=0.32 \frac{\eta_{\text {surf }}}{\eta_{\text {sol }}}$ and $\frac{\sigma_{s}}{\sigma_{\text {sol }}}=0.07 \frac{\eta_{\text {surf }}}{\eta_{\text {sol }}}$, to be compared with the experimental values $\frac{\sigma_{p}}{\sigma_{\text {sol }}}=0.18$ and $\frac{\sigma_{s}}{\sigma_{\text {sol }}}=0.04$. In either case, one finds $\frac{\eta_{\text {surf }}}{\eta_{\text {sol }}} \approx 0.55$. Similarly, for the Vis transition, the computed values are $\frac{\sigma_{p}}{\sigma_{\text {sol }}}=0.66 \frac{\eta_{\text {surf }}}{\eta_{\text {sol }}}$ and $\frac{\sigma_{s}}{\sigma_{\text {sol }}}=$ $0.15 \frac{\eta_{\text {surf }}}{\eta_{\text {sol }}}$, to be compared with the experimental values $\frac{\sigma_{p}}{\sigma_{\text {sol }}}=0.37$ and $\frac{\sigma_{s}}{\sigma_{\text {sol }}}=0.08$. Once again, in either case, one finds $\frac{\eta_{s u r f}}{\eta_{s o l}} \approx 0.55$. Therefore, within the framework of this quantitative analysis, the isomerization quantum yield is found to be independent on light 
polarization, which appears reasonable since $\eta$ is expected to depend mostly on the properties of the transition state and the coupling of the molecule to its environment, both independent on the photon field. For both the UV and Vis transitions, the $\frac{\eta_{\text {surf }}}{\eta_{\text {sol }}}$ ratio is found to exhibit nearly the same value of 0.55 , which can be paralleled to the closely similar configuration of $\mathrm{C}$ and $\mathrm{E}$ isomers at the surface. It corresponds to an isomerization quantum yield about twice less efficient at the surface than in solution.

The origin of this difference cannot be sought for in terms of local electric field (already taken into account in the above analysis), steric effect between neighbour molecules (since the experimental cross sections do not significantly depend on the surface molecule concentration), or electronic interaction with the substrate (shielded by the molecular layer grafted at the surface). Therefore, we tentatively assign the loss of efficiency of the isomerization at surfaces to the interaction of fulgimide molecules with the surface molecular layer grafted on silicon, which makes them adopt the tilted configuration determined in Section 4.1.

\section{Conclusion}

In conclusion, the present results demonstrate that when photochromic molecules are immobilized at a surface in a well-controlled configuration, a quantitative analysis of the photoswitching kinetics allows for obtaining a detailed picture of the system at the molecular level. In the particular case of indolyl fulgimide compounds covalently anchored on functionalized $\mathrm{Si}(111)$ surfaces the data above show that the photoisomerization remains efficient and reversible and that the photo stationary compositions are close to those found in solution provided steric hindrance is avoided by limiting the surface density of immobilized photochromic molecules, here below 0.9 isomers $/ \mathrm{nm}^{2}$. 
However, from a dynamic view point, surface photokinetics strongly depart from that in solution. The photoisomerization cross sections measured at the surface are much smaller than in solution. In addition, the photokinetics is no longer following a pure first order law. Only a fraction of the photochromic molecules switches with a constant photoisomerization cross section $\sigma$. Above a critical threshold, $\sigma$ continuously decreases with increasing photon dose. The initial $\sigma$ is representative of the isomers which can freely react upon light absorption and measurements under s- and p-polarized light indicate that the transition dipole of the switching isomers is almost lying in the surface plane, a configuration which corresponds to fulgimides partially embedded in the underlying monolayer. Noteworthy, the open (E) and closed (C) forms exhibit similar configuration. This configuration is likely at the origin of the reduced value of the isomerization quantum yield $\eta$ which can be derived when comparing experimental photo-isomerization cross sections recorded for "freely" switchable molecules at the surface with those measured in solution.

\section{Appendix}

It is worth recalling that typical photochromic reactions $\mathrm{A} \leftrightarrow \mathrm{B}$ follow the general photokinetic differential equations [76] involving concentrations of all isomers, their related absorption coefficients, thermal reaction rates and photochromic quantum yields corresponding to $\mathrm{A} \rightarrow \mathrm{B}$ and $\mathrm{B} \rightarrow \mathrm{A}$ reactions. In the general case and in absence of any approximation, these differential equations show nonlinear terms (known as photokinetic factors) preventing any analytical solution. Nevertheless, in the case of the present fulgimide molecule, thermal reaction rates can be neglected and concentrations are extremely low: absorbance values of our samples at the irradiation wavelengths are smaller than 0.1 in solution and 0.001 at the surface, especially for surfaces functionalized with a (diluted) monolayer of photo-active 
molecules. Consequently, photochromic differential equations linearization gives simple first order photokinetics.

However, when photoswitching strongly deviates from a pure exponential law, it appears difficult to make a quantitative comparison between different configurations. To this end, a procedure described below was used to determine the isomeric composition at PSS and the isomerization cross section $\sigma$ from partial photoconversion experiments.

Figure A1 is an example of such a full data analysis. In top panels the solid black lines are plots of the advancement of the Vis and then UV isomerization processes of an initially pure $\mathrm{C}$ monolayer after calibration of the $\mathrm{y}$-scale. Data are plotted as function of the logarithm of the photon dose $p$. The relevance of this plot is justified by Fig. S5. The calibration of the y-scale was explained for Fig. 1. The derivative of the plots (blue crosses) is empirically fitted to a Voigt function (red line); integration of the Voigt function gives the expected advancement of the photo isomerization, which obviously superimposes the solid black line but also extends it at photon doses for which photochromism was not measured (black dashed line). Such an extrapolation yields the isomeric composition at PSS. This analysis immediately captures that photoconversion was (voluntary made) incomplete, because $p$ was too small to reach the PSSs. This also evidences that a fraction of the $\mathrm{C}$-isomers remains blocked (the monolayer density is $1.6 / \mathrm{nm}^{2}$ which is above the maximum packing density of isomers in 2D). Panel (b) of Fig. A1, is a plot of the cross section which is obtained as $\sigma=\frac{1}{[C(p)]} \frac{\partial[C(p)]}{\partial p}$, with $[C(p)]$ the surface density of switched isomers after illumination with a photon dose $p$. This plot outlines that $\sigma$ is constant up to a critical photon dose $p^{*}$ (regime featured by grey shaded regions) above which a progressive decrease of $\sigma$ is observed. The initial $\sigma$ (i.e., for $\left.p<p^{*}\right)$ is accurately obtained by replotting the data as in Fig. 4 where $\ln (1-$ $\mathrm{E} / \mathrm{E}_{\mathrm{PSS}}$ ) is plotted against $p$ (PSS is the above determined value). Linear regression restricted to $p<p^{*}$ yields $\sigma$ with no ambiguity. 
The back photo isomerization (panels $\mathrm{c}$ and $\mathrm{d}$ ) was analysed by the same method. However, it is important to note that the situation under UV irradiation is intrinsically more complicated because several photoreactions are expected to occur: $\mathrm{E} \rightarrow \mathrm{C}$, but also $\mathrm{C} \rightarrow \mathrm{E}$, $\mathrm{E} \rightarrow \mathrm{Z}$ and $\mathrm{Z} \rightarrow \mathrm{E}$. As a consequence, the $\sigma_{\mathrm{UV}}$ values do not correspond strictly to the single $\mathrm{E} \rightarrow \mathrm{C}$ isomerization. In initial stages of UV irradiation, starting from a E-terminated monolayer, and the $\mathrm{E} \rightarrow \mathrm{C}$ and $\mathrm{E} \rightarrow \mathrm{Z}$ reactions represent the major pathways. Under prolonged irradiation, the $\mathrm{C} \rightarrow \mathrm{E}$ and $\mathrm{Z} \rightarrow \mathrm{E}$ may play an important role in the system evolution. This time-dependent behaviour may be partly responsible for the decrease of $\sigma$ as a function of UV photon dose, and the lower value of $p^{*}$ compared to the one determined under visible illumination. However, at low photon doses, the $\sigma_{\mathrm{UV}}$ value can be approximated to be mostly representative of the $\mathrm{E} \rightarrow \mathrm{C}$ reaction because (i) the $\mathrm{E} \leftrightarrow \mathrm{Z}$ photoisomerizations play a negligible role (there are only few percent of $\mathrm{Z}$ forms in the PSS composition) and (ii) the $\mathrm{C}$ form concentration is very low at initial stage.

It should be noted that the procedure above was tested over a large number of experiments and appears robust. The very same behaviour was indeed observed for all photoconversion experiments for all samples, regardless the surface density of the monolayer. In particular, we emphasize that for the stabilization of the fitting procedure, the fitting with the pseudo Voigt function was performed keeping fixed the Lorentzian and Gaussian width and a fixed Gaussian/Lorentzian ratio for all experiments. 

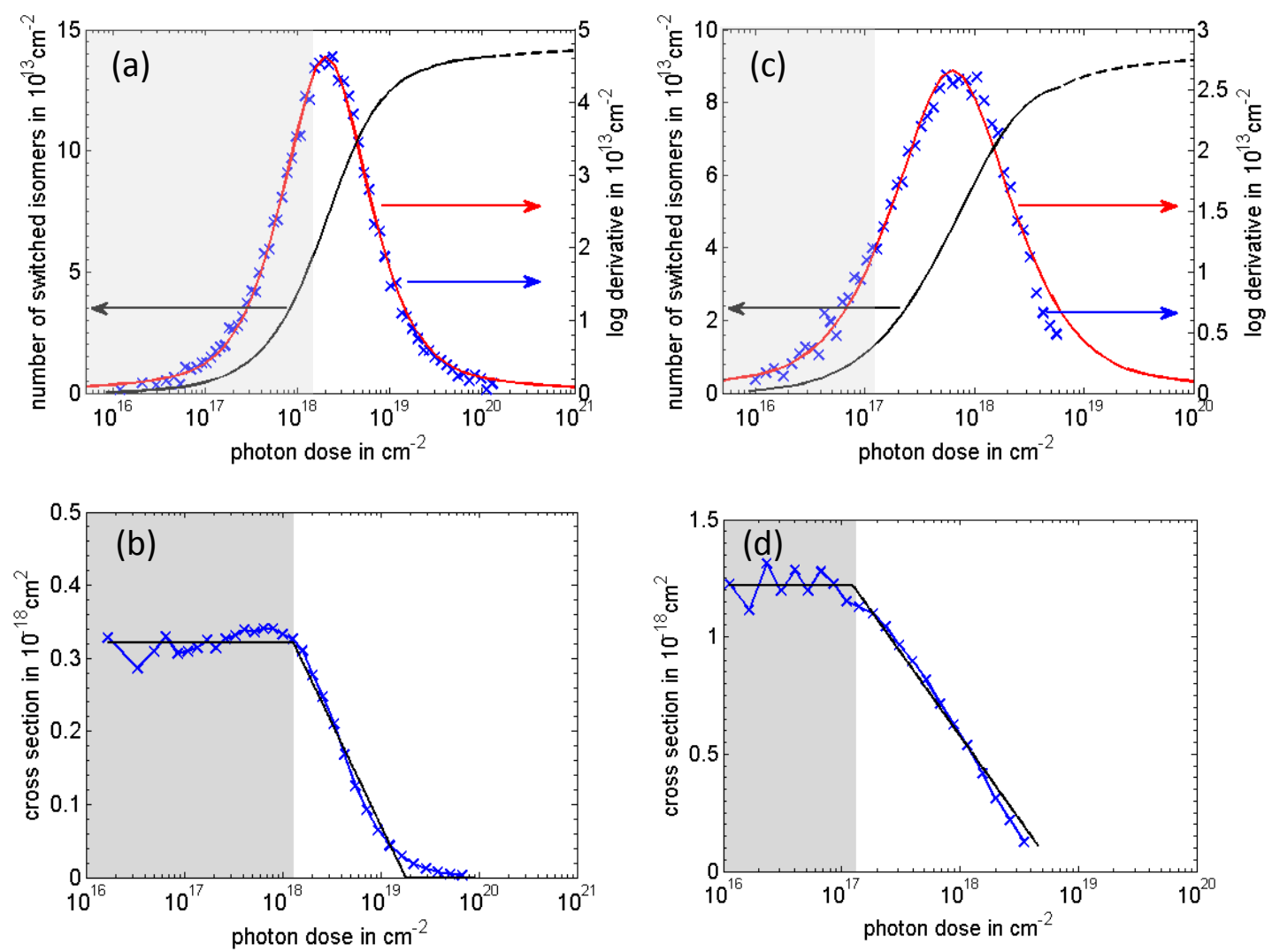

Fig. A1: Example of data analysis starting for a pure C-monolayer with 1.6 isomers $/ \mathrm{nm}^{2}$ exposed to visible illumination (left column) and then to UV illumination (right column). This analysis corresponds to the data presented in Fig. 1: (a) and (c) the black solid and dashed lines correspond to experimental and calculated variations of the number of switched isomers, respectively, as a function of $\log (p)$, with $\mathrm{p}$ the photon dose. Blue symbols are the derivative of the black line and the red line is the best fit using a Voigt function; (b) and (d) variations of the numerical cross section as a function of $\log (p)$. Notice the plateau below a critical value $p^{*}$ (grey shaded regions).

\section{Acknowledgements}

This work has been supported by a public grant overseen by the French National Research Agency (ANR) as part of the "Investissements d'Avenir" program (Labex charmmmat, ANR11-LABX-0039-grant). We also thank Technische Universität and the Deutsche Forschungsgemeinschaft (SFB 658) for financial support. 


\section{Famerican}

\section{References}

[1] H. Bouas-Laurent and H. Dürr, Organic photochromism, Pure Appl. Chem. 73, 639 (2001).

[2] G. S. Kumar and D. C. Neckers, Photochemistry of azobenzene-containing polymers, Chem. Rev. 89, 1915 (1989).

[3] A. Natansohn and P. Rochon, Photoinduced Motions in Azo-Containing Polymers, Chemical Reviews 102, 4139 (2002).

[4] S. Yagai and A. Kitamura, Recent advances in photoresponsive supramolecular selfassemblies, Chem. Soc. Rev. 37, 1520 (2008).

[5] R. Pardo, M. Zayat, and D. Levy, Photochromic organic-inorganic hybrid materials, Chem. Soc. Rev. 40, 672 (2011).

[6] P. Rochon, E. Batalla, and A. Natansohn, Optically induced surface gratings on azoaromatic polymer films, Appl. Phys. Lett. 66, 136 (1995).

[7] H. Finkelmann, E. Nishikawa, G. G. Pereira, and M. Warner, A New Opto-Mechanical Effect in Solids, Physical Review Letters 87, 015501 (2001).

[8] K. E. Maly, M. D. Wand, and R. P. Lemieux, Bistable Ferroelectric Liquid Crystal Photoswitch Triggered by a Dithienylethene Dopant, Journal of the American Chemical Society 124, 7898 (2002).

[9] Y. Yu, M. Nakano, and T. Ikeda, Photomechanics: Directed bending of a polymer film by light, Nature 425, 145 (2003).

[10] F. Fabbri, Y. Lassailly, K. Lahlil, J. P. Boilot, and J. Peretti, Alternating photoinduced mass transport triggered by light polarization in azobenzene containing sol-gel films, Appl. Phys. Lett. 96, 081908 (2010).

[11] Z. F. Liu, K. Hashimoto, and A. Fujishima, Photoelectrochemical information storage using an azobenzene derivative, Nature 347, 658 (1990).

[12] S. Rath, M. Heilig, H. Port, and J. Wrachtrup, Periodic Organic Nanodot Patterns for Optical Memory, Nano Lett. 7, 3845 (2007).

[13] Y. C. Liang, A. S. Dvornikov, and P. M. Rentzepis, Nonvolatile read-out molecular memory, Proceedings of the National Academy of Sciences 100, 8109 (2003).

[14] M. Sense Jan van der and L. Peter, Charge transport through molecular switches, Journal of Physics: Condensed Matter 22, 133001 (2010).

[15] H. Zhang, X. Guo, J. Hui, S. Hu, W. Xu, and D. Zhu, Interface Engineering of Semiconductor/Dielectric Heterojunctions toward Functional Organic Thin-Film Transistors, Nano Lett. 11, 4939 (2011).

[16] W. Szymański, J. M. Beierle, H. A. V. Kistemaker, W. A. Velema, and B. L. Feringa, Reversible Photocontrol of Biological Systems by the Incorporation of Molecular Photoswitches, Chemical Reviews 113, 6114 (2013).

[17] I. Willner and S. Rubin, Control of the Structure and Functions of Biomaterials by Light, Angewandte Chemie International Edition in English 35, 367 (1996).

[18] T. Arif, C. Cazorla, N. Bogliotti, N. Saleh, F. Blanchard, V. Gandon, R. Métivier, J. Xie, A. Voituriez, and A. Marinetti, Bimetallic gold(i) complexes of photoswitchable phosphines: synthesis and uses in cooperative catalysis, Catalysis Science \& Technology 8, 710 (2018).

[19] U. Jung, O. Filinova, S. Kuhn, D. Zargarani, C. Bornholdt, R. Herges, and O. Magnussen, Photoswitching Behavior of Azobenzene-Containing Alkanethiol SelfAssembled Monolayers on Au Surfaces, Langmuir 26, 13913 (2010). 
[20] Y. Wen, W. Yi, L. Meng, M. Feng, G. Jiang, W. Yuan, Y. Zhang, H. Gao, L. Jiang, and Y. Song, Photochemical-Controlled Switching Based on Azobenzene Monolayer Modified Silicon (111) Surface, J. Phys. Chem. B 109, 14465 (2005).

[21] K. Rück-Braun, M. Å. Petersen, F. Michalik, A. Hebert, D. Przyrembel, C. Weber, S. A. Ahmed, S. Kowarik, and M. Weinelt, Formation of Carboxy- and Amide-Terminated Alkyl Monolayers on Silicon(111) Investigated by ATR-FTIR, XPS, and X-ray Scattering: Construction of Photoswitchable Surfaces, Langmuir 29, 11758 (2013).

[22] C. Henry de Villeneuve, F. Michalik, J. N. Chazalviel, K. Rück-Braun, and P. Allongue, Quantitative IR Readout of Fulgimide Monolayer Switching on Si(111) Surfaces, Advanced Materials 25, 416 (2013).

[23] S. D. Evans, S. R. Johnson, H. Ringsdorf, L. M. Williams, and H. Wolf, Photoswitching of Azobenzene Derivatives Formed on Planar and Colloidal Gold Surfaces, Langmuir 14, 6436 (1998).

[24] M. El Garah, F. Palmino, and F. Cherioux, Reversible Photoswitching of AzobenzeneBased Monolayers Physisorbed on a Mica Surface, Langmuir 26, 943 (2009).

[25] S. Michael and H. Marcus, Photoactive self-assembled monolayers for optically switchable organic thin-film transistors, Appl. Phys. Lett. 102, 203301 (2013).

[26] F. Nickel, M. Bernien, K. Kraffert, D. Krüger, L. M. Arruda, L. Kipgen, and W. Kuch, Reversible Switching of Spiropyran Molecules in Direct Contact With a Bi(111) Single Crystal Surface, Advanced Functional Materials 27, 1702280 (2017).

[27] S. V. Snegir, A. A. Marchenko, P. Yu, F. Maurel, O. L. Kapitanchuk, S. Mazerat, M. Lepeltier, A. Leaustic, and E. Lacaze, STM Observation of Open- and Closed-Ring Forms of Functionalized Diarylethene Molecules Self-Assembled on a $\mathrm{Au}(111)$ Surface, J. Phys. Chem. Lett. 2, 2433 (2011).

[28] R. Wang, L. Jiang, T. Iyoda, D. A. Tryk, K. Hashimoto, and A. Fujishima, Investigation of the Surface Morphology and Photoisomerization of an Azobenzene-Containing Ultrathin Film, Langmuir 12, 2052 (1996).

[29] K. Ichimura, S.-K. Oh, and M. Nakagawa, Light-Driven Motion of Liquids on a Photoresponsive Surface, Science 288, 1624 (2000).

[30] K. Smaali, S. Lenfant, S. Karpe, M. Oçiafrain, P. Blanchard, D. Deresmes, S. Godey, A. Rochefort, J. Roncali, and D. Vuillaume, High On-Off Conductance Switching Ratio in Optically-Driven Self-Assembled Conjugated Molecular Systems, ACS Nano 4, 2411 (2010).

[31] K. Uchida, Y. Yamanoi, T. Yonezawa, and H. Nishihara, Reversible On/Off Conductance Switching of Single Diarylethene Immobilized on a Silicon Surface, J. Am. Chem. Soc. 133, 9239 (2011).

[32] N. Crivillers, E. Orgiu, F. Reinders, M. Mayor, and P. Samorì, Optical Modulation of the Charge Injection in an Organic Field-Effect Transistor Based on Photochromic SelfAssembled-Monolayer-Functionalized Electrodes, Adv. Mater. 23, 1447 (2011).

[33] T. Masuda, M. Irie, and K. Uosaki, Photoswitching of electron transfer property of diarylethene viologen linked molecular layer constructed on a hydrogen-terminated Si(111) Surface, Thin Solid Films 518, 591 (2009).

[34] X. Zhang, Y. Wen, Y. Li, G. Li, S. Du, H. Guo, L. Yang, L. Jiang, H. Gao, and Y. Song, Molecularly Controlled Modulation of Conductance on Azobenzene MonolayerModified Silicon Surfaces, The Journal of Physical Chemistry C 112, 8288 (2008).

[35] A. Yoshihiro, N. Hikaru, K. Masami, N. Yoshitaka, L. Yan Jun, and S. Yasuhiro, Switching surface polarization of atomic force microscopy probe utilizing photoisomerization of photochromic molecules, Journal of Applied Physics 109, 064308 (2011). 
[36] M. J. Comstock, N. Levy, A. Kirakosian, J. Cho, F. Lauterwasser, J. H. Harvey, D. A. Strubbe, J. M. J. Fréchet, D. Trauner, S. G. Louie, and M. F. Crommie, Reversible Photomechanical Switching of Individual Engineered Molecules at a Metallic Surface, Physical Review Letters 99, 038301 (2007).

[37] A. S. Kumar, T. Ye, T. Takami, B.-C. Yu, A. K. Flatt, J. M. Tour, and P. S. Weiss, Reversible Photo-Switching of Single Azobenzene Molecules in Controlled Nanoscale Environments, Nano Lett. 8, 1644 (2008).

[38] W. Bronsch, D. Przyrembel, L. Boie, C. Gahl, and M. Weinelt, Fast optical workfunction tuning at an organic/metal interface, Appl. Phys. Lett. 111, 081601 (2017).

[39] R. Klajn, J. F. Stoddart, and B. A. Grzybowski, Nanoparticles functionalised with reversible molecular and supramolecular switches, Chem. Soc. Rev. 39, 2203 (2010).

[40] T. Petra, Optically and thermally induced molecular switching processes at metal surfaces, Journal of Physics: Condensed Matter 24, 394001 (2012).

[41] H. M. D. Bandara and S. C. Burdette, Photoisomerization in different classes of azobenzene, Chemical Society Reviews 41, 1809 (2012).

[42] T. Nägele, R. Hoche, W. Zinth, and J. Wachtveitl, Femtosecond photoisomerization of cis-azobenzene, Chemical Physics Letters 272, 489 (1997).

[43] I. K. Lednev, T. Q. Ye, P. Matousek, M. Towrie, P. Foggi, F. V. R. Neuwahl, S. Umapathy, R. E. Hester, and J. N. Moore, Femtosecond time-resolved UV-visible absorption spectroscopy of trans-azobenzene: dependence on excitation wavelength, Chemical Physics Letters 290, 68 (1998).

[44] B. G. Levine and T. J. Martínez, Isomerization Through Conical Intersections, Annual Review of Physical Chemistry 58, 613 (2007).

[45] Y. Yokoyama, Fulgides for Memories and Switches, Chem. Rev. 100, 1717 (2000).

[46] S. Draxler, T. Brust, S. Malkmus, F. O. Koller, B. Heinz, S. Laimgruber, C. Schulz, S. Dietrich, K. Rück-Braun, W. Zinth, and M. Braun, Ultrafast reaction dynamics of the complete photo cycle of an indolylfulgimide studied by absorption, fluorescence and vibrational spectroscopy, Journal of Molecular Liquids 141, 130 (2008).

[47] S. Malkmus, F. O. Koller, B. Heinz, W. J. Schreier, T. E. Schrader, W. Zinth, C. Schulz, S. Dietrich, K. Rück-Braun, and M. Braun, Ultrafast ring opening reaction of a photochromic indolyl-fulgimide, Chem. Phys. Lett. 417, 266 (2006).

[48] F. O. Koller, W. J. Schreier, T. E. Schrader, S. Malkmus, C. Schulz, S. Dietrich, K. Ruck-Braun, and M. Braun, Ultrafast Ring-Closure Reaction of Photochromic Indolylfulgimides Studied with UV-pump-IR-Probe Spectroscopy, J. Phys. Chem. A 112, 210 (2007).

[49] G. Berkovic, V. Krongauz, and V. Weiss, Spiropyrans and Spirooxazines for Memories and Switches, Chem. Rev. 100, 1741 (2000).

[50] H. Gorner, Photochromism of nitrospiropyrans: effects of structure, solvent and temperature, Phys. Chem. Chem. Phys. 3, 416 (2001).

[51] J. Kohl-Landgraf, M. Braun, C. Özçoban, D. P. N. Gonçalves, A. Heckel, and J. Wachtveitl, Ultrafast Dynamics of a Spiropyran in Water, Journal of the American Chemical Society 134, 14070 (2012).

[52] M. Irie, Diarylethenes for Memories and Switches, Chem. Rev. 100, 1685 (2000).

[53] H. Tian and S. Yang, Recent progresses on diarylethene based photochromic switches, Chemical Society Reviews 33, 85 (2004).

[54] J. Henzl, T. Bredow, and K. Morgenstern, Irreversible isomerization of the azobenzene derivate Methyl Orange on Au(111), Chemical Physics Letters 435, 278 (2007).

[55] K. M. McElhinny, J. Park, Y. Ahn, P. Huang, Y. Joo, A. Lakkham, A. Pateras, H. Wen, P. Gopalan, and P. G. Evans, Photoisomerization Dynamics in a Densely Packed Optically Transformable Azobenzene Monolayer, Langmuir 34, 10828 (2018). 
[56] X. Pei, A. Fernandes, B. Mathy, X. Laloyaux, B. Nysten, O. Riant, and A. M. Jonas, Correlation between the Structure and Wettability of Photoswitchable Hydrophilic Azobenzene Monolayers on Silicon, Langmuir 27, 9403 (2011).

[57] B. Baisch, D. Raffa, U. Jung, O. M. Magnussen, C. Nicolas, J. Lacour, J. Kubitschke, and R. Herges, Mounting Freestanding Molecular Functions onto Surfaces: The Platform Approach, J. Am. Chem. Soc. 131, 442 (2008).

[58] U. Jung, S. Kuhn, U. Cornelissen, F. Tuczek, T. Strunskus, V. Zaporojtchenko, J. Kubitschke, R. Herges, and O. Magnussen, Azobenzene-Containing Triazatriangulenium Adlayers on $\mathrm{Au}(111)$ : Structural and Spectroscopic Characterization, Langmuir 27, 5899 (2011).

[59] P. Dietrich, F. Michalik, R. Schmidt, C. Gahl, G. Mao, M. Breusing, M. Raschke, B. Priewisch, T. Elsässer, R. Mendelsohn, M. Weinelt, and K. Rück-Braun, An anchoring strategy for photoswitchable biosensor technology: azobenzene-modified SAMs on Si(111), Appl. Phys. A-Mater 93, 285 (2008).

[60] M. Schulze, M. Utecht, T. Moldt, D. Przyrembel, C. Gahl, M. Weinelt, P. Saalfrank, and P. Tegeder, Nonlinear optical response of photochromic azobenzene-functionalized self-assembled monolayers, Phys. Chem. Chem. Phys. 17, 18079 (2015).

[61] T. Moldt, D. Przyrembel, M. Schulze, W. Bronsch, L. Boie, D. Brete, C. Gahl, R. Klajn, P. Tegeder, and M. Weinelt, Differing Isomerization Kinetics of AzobenzeneFunctionalized Self-Assembled Monolayers in Ambient Air and in Vacuum, Langmuir 32, 10795 (2016).

[62] X. L. Zhou, X. Y. Zhu, and J. M. White, Photochemistry at adsorbate/metal interfaces, Surface Science Reports 13, 73 (1991).

[63] M. L. Munford, R. Cortès, and P. Allongue, The preparation of ideally ordered flat HSi(111) surfaces, Sensor. Mater. 13, 259 (2001).

[64] X. Wallart, C. Henry de Villeneuve, and P. Allongue, Truly quantitative XPS characterization of organic monolayers on silicon: Study of alkyl and alkoxy monolayers on H-Si(111), J. Am. Chem. Soc. 127, 7871 (2005).

[65] A. Faucheux, A. C. Gouget-Laemmel, C. H. de Villeneuve, R. Boukherroub, F. Ozanam, P. Allongue, and J. N. Chazalviel, Well-defined carboxyl-terminated alkyl monolayers grafted onto H-Si(111): Packing density from a combined AFM and quantitative IR study, Langmuir 22, 153 (2006).

[66] A. Moraillon, A. C. Gouget-Laemmel, F. Ozanam, and J.-N. Chazalviel, Amidation of Monolayers on Silicon in Physiological Buffers: A Quantitative IR Study, J. Phys. Chem. C 112, 7158 (2008).

[67] A. D. Becke, Density-functional thermochemistry. III. The role of exact exchange, The Journal of Chemical Physics 98, 5648 (1993).

[68] G. W. T. M. J. Frisch, H. B. Schlegel, G. E. Scuseria, M. A. Robb, J. R. Cheeseman, G. Scalmani, V. Barone, G. A. Petersson, H. Nakatsuji, X. Li, M. Caricato, A. Marenich, J. Bloino, B. G. Janesko, R. Lipparini, F. Egidi, J. Goings, B. Peng, A. Petrone, T. Henderson, D. Ranasinghe, V. G. Zakrzewski, J. Gao, N. Rega, G. Zheng, W. Liang, M. Hada, M. Ehara, K. Toyota, R. Fukuda, J. Hasegawa, M. Ishida, T. Nakajima, Y. Honda, O. Kitao, H. Nakai, T. Vreven, K. Throssell, J. A. Montgomery, J. E. Peralta, F. Ogliaro, M. Bearpark, J. J. Heyd, E. Brothers, K. N. Kudin, V. N. Staroverov, T. Keith, R. Kobayashi, J. Normand, K. Raghavachari, A. Rendelll, J. C. Burant, S. S. Iyengar, J. Tomasi, M. Cossi, J. M. Millam, M. Klene, C. Adamo, R. Cammi, J. W. Ochterski, R. L. Martin, K. Morokuma, O. Farkas, J. B. Foresman and D. J. Fox,, Gaussian 09, Revision A.02, Gaussian, Inc., Wallington CT, 2016. 
[69] A. B. Sieval, B. van den Hout, H. Zuilhof, and E. J. R. Sudhölter, Molecular Modeling of Covalently Attached Alkyl Monolayers on the Hydrogen-Terminated Si(111) Surface, Langmuir 17, 2172 (2001).

[70] V. Deblauwe and G. Smets, Quantum yields of the photochromic reactions of heterocyclic fulgides and fulgimides, Die Makromolekulare Chemie 189, 2503 (1988).

[71] M. Schulze, M. Utecht, A. Hebert, K. Rück-Braun, P. Saalfrank, and P. Tegeder, Reversible Photoswitching of the Interfacial Nonlinear Optical Response, The Journal of Physical Chemistry Letters 6, 505 (2015).

[72] S. R. Polo and M. K. Wilson, Infrared Intensities in Liquid and Gas Phases, The Journal of Chemical Physics 23, 2376 (1955).

[73] A. Klaus, B. Richard, E. Manfred, and W. J. H., Laser-induced reorientation of the optical axis in liquid-crystalline side chain polymers, Die Makromolekulare Chemie, Rapid Communications 10, 477 (1989).

[74] Y. J. Chabal, Surface infrared spectroscopy, Surface Science Reports 8, 211 (1988).

[75] D. F. Edwards, in Handbook of Optical Constants of Solids, edited by E. D. Palik (Academic Press, Burlington, 1997), pp. 547.

[76] J. P. K. Nakatani, P. Yu, R. Métivier, Introduction: organic photochromic molecules, in Photochromic materials: preparation, properties and applications, 1st Edition, Tian H., Zhang J. (eds.), Wiley-VCH, 2016, pp. 1-45 (ISBN: 978-3-527-33779-8). 


\section{Tables:}

\section{Table 1:}

PSS composition given as $\mathrm{C} / \mathrm{E}+\mathrm{Z}$ relative proportions, in solution (acetonitrile) and at surfaces. $\boldsymbol{D}$ is the initial fulgimide concentration at the surface and $\boldsymbol{D}^{*} \sim 0.9$ isomers $/ \mathrm{nm}^{2}$ is the density below which no steric hindrance occurs (see text).

\begin{tabular}{|l|c|c|c|}
\hline & Solution & $\begin{array}{c}\text { Surface } \\
\left(\boldsymbol{D} \leq \boldsymbol{D}^{*}\right)\end{array}$ & $\begin{array}{c}\text { Surface } \\
\left(\boldsymbol{D}>\boldsymbol{D}^{*}\right)\end{array}$ \\
\hline Visible (525 nm) & $0 / 100$ & $0 / 100$ & $8 / 92$ \\
\hline UV (365 nm) & $77 / 23^{\text {a) }}$ & $66 / 34$ & $63 / 37$ \\
\hline
\end{tabular}

a) This composition is in agreement with literature $(\mathrm{C} / \mathrm{E} / \mathrm{Z})=(77 / 21 / 2)[22][48]$

\section{Table 2:}

Comparison of isomerization cross sections measured at surfaces (left block) and in solution (right block). For the surface light is either non-polarized, p-pol. and s-pol. light and in solution (right block). All cross sections are given in units of $10^{-18} \mathrm{~cm}^{2}$. UV and Vis wavelengths are $365 \mathrm{~nm}$ and $525 \mathrm{~nm}$ respectively. Note that $\sigma$-values at surfaces are quasiindependent of the fulgimide density.

\begin{tabular}{|c|c|c|c|c|c|c|c|c|}
\hline \multirow{3}{*}{$\frac{\text { Polarization }}{\lambda}$} & \multicolumn{6}{|c|}{ surface } & \multirow{2}{*}{\multicolumn{2}{|c|}{$\begin{array}{c}\text { solution } \\
\text { na } \\
\end{array}$}} \\
\hline & \multicolumn{2}{|c|}{ non-pol. } & \multicolumn{2}{|c|}{ p-pol. } & \multicolumn{2}{|c|}{ s-pol. } & & \\
\hline & $\mathbf{U V}$ & Vis & $\mathbf{U V}$ & Vis & UV & Vis & $\mathbf{U V}$ & Vis \\
\hline$\sigma_{\text {Vis }}$ & & $\begin{array}{c}0.33 \pm \\
0.01\end{array}$ & - & $0.40 \pm 0.06$ & - & $0.09 \pm 0.03$ & - & $1.08 \pm 0.04$ \\
\hline$\sigma_{\mathbf{U V}}$ & $\begin{array}{c}1.30 \pm \\
0.05\end{array}$ & & $\begin{array}{c}1.51 \pm \\
0.11 \\
\end{array}$ & - & $\begin{array}{c}0.35 \pm \\
0.04\end{array}$ & - & $\begin{array}{c}8.31 \pm \\
0.29 \\
\end{array}$ & - \\
\hline$\sigma_{\mathrm{Abs}}$ & & & & & & & 30.3 & 16.4 \\
\hline$\eta$ & & & & & & & 0.27 & 0.066 \\
\hline
\end{tabular}




\section{Schemes:}

C

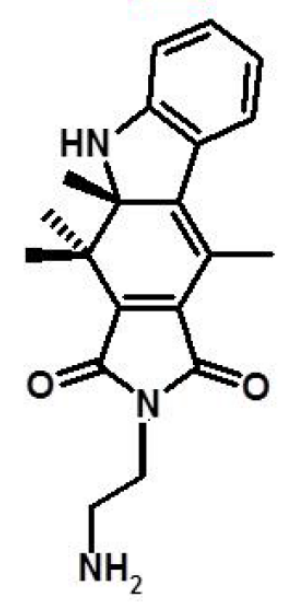

E
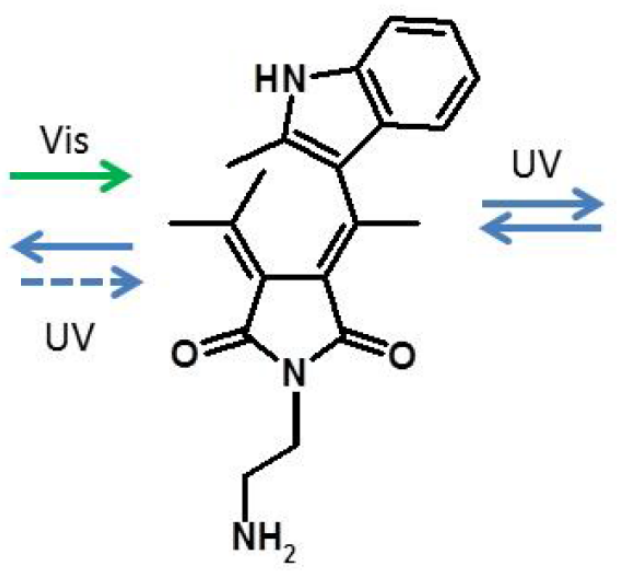

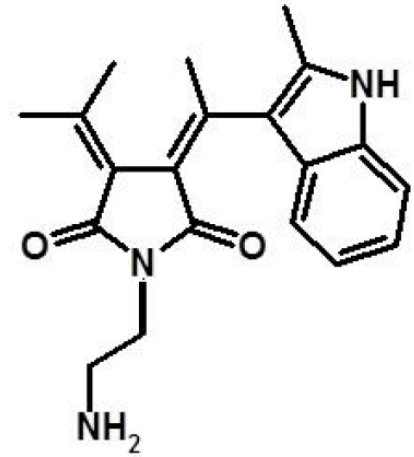

\section{Scheme 1:}

Molecular structure of indolyl fulgimide isomers. Solid arrows represent the photochromic reactions occuring under visible $(\lambda=525 \mathrm{~nm})$ and UV light $(\lambda=365 \mathrm{~nm})$. The dashed arrow is accounting for the PSS composition under UV (see text).

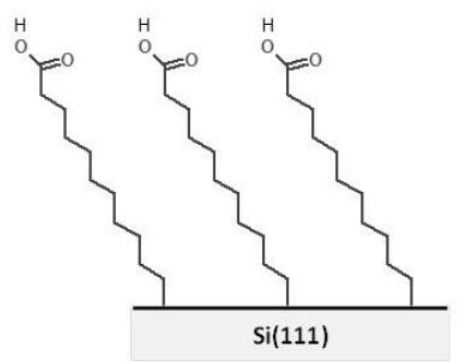

(a)

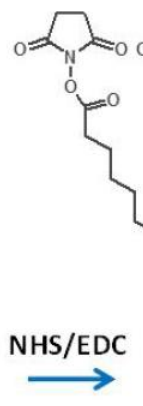

\section{Scheme 2:}

Schematic description of the multistep protocol used to immobilize fulgimide compounds on Si(111). (a) Alkyl monolayer terminated by carboxylic acid groups prepared according to Ref. [65]; (b) A succinimidyl-ester "activated" surface is obtained by exposure of (a) to a NHS/EDC aqueous solution; (c) Fulgimide monolayer: variable densities of photochromic molecules are obtained by exposing the "activated" surfaces to a mixture of amino-ethyl indolyl fulgimide and ethyl amine in acetonitrile (see experimental details). 


\section{Figures:}
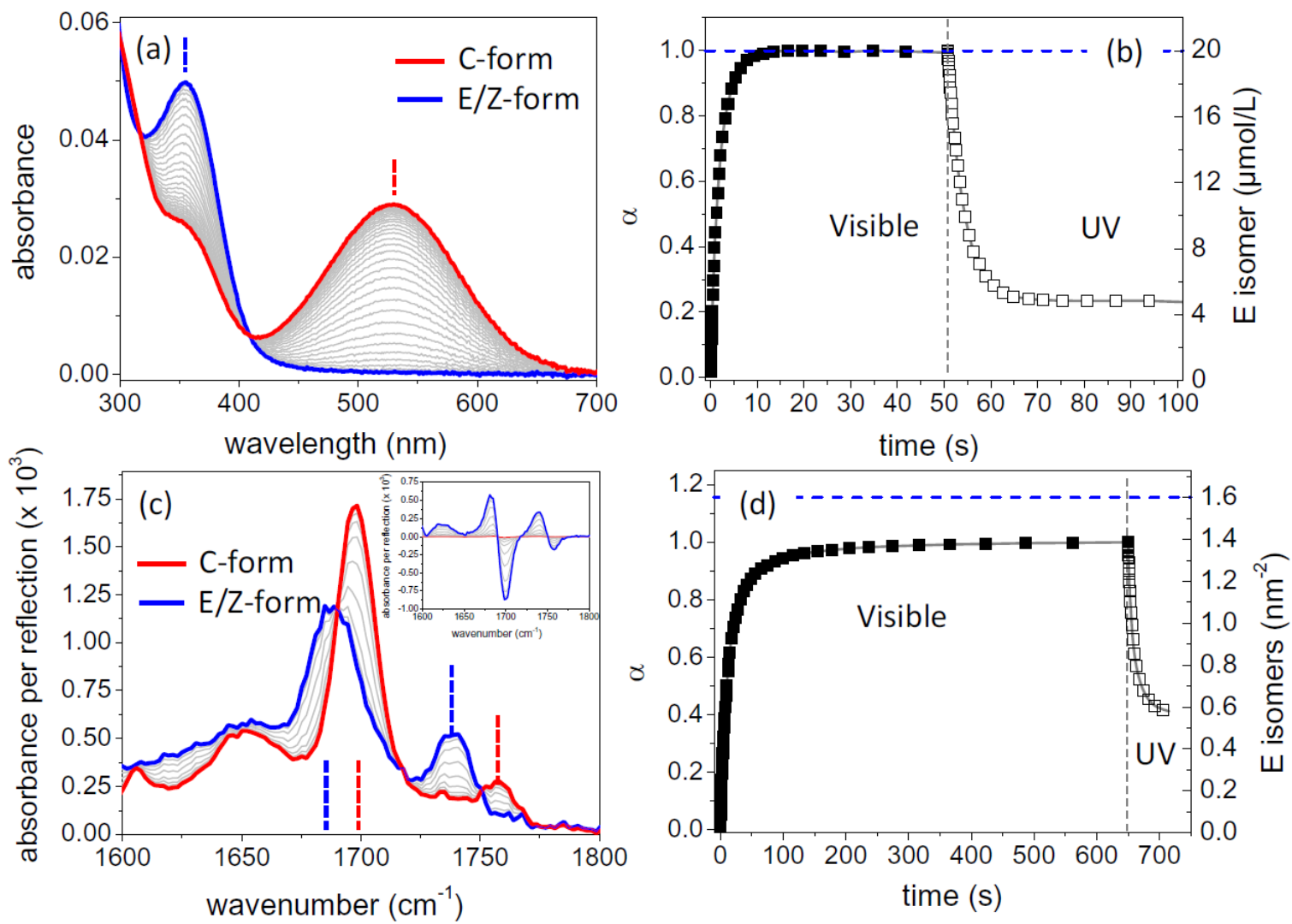

\section{Figure 1:}

Comparison of photochromism in solution (a-b) and at surface (c-d). Initial state is a pure Csolution or a pure C-monolayer. (a) Series of UV-Vis spectra recorded during the Vis illumination of a $20 \mu \mathrm{M} \mathrm{C}$-isomers solution in acetonitrile. The initial spectrum (red line) is that of the pure $\mathrm{C}$ isomers. The blue line is the last spectrum of the experiment and is very close to that of pure E-solution. (b) Time dependence of relative advancement yield $\alpha$ (left scale), along the first Vis and subsequent UV irradiation periods. (c) Series of narrow FTIR spectra recorded during the Vis illumination of a pure $\mathrm{C}$ monolayer, with 1.6 fulgimides $/ \mathrm{nm}^{2}$. The initial spectrum (red line) is that of the pure C-terminated surface. Note that the blue line (last spectrum) is close to that of a pure E-terminated surface. (d) Same as (b). In panels (b) and (d), quantitative solution and surface compositions are given by the right y-scale (see text for more explanation). The concentration of isomers in solution and their density on the surface are highlighted with horizontal blue dashed lines in (b) and (d). The vertical dashed line marks the transition from Vis to UV illumination. 
(a)

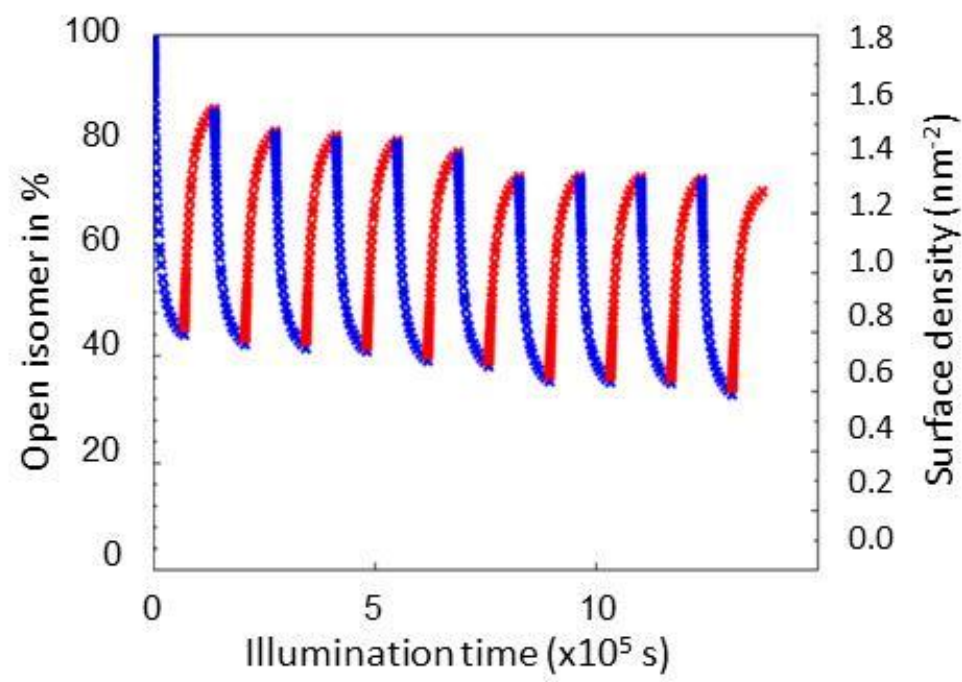

(b)

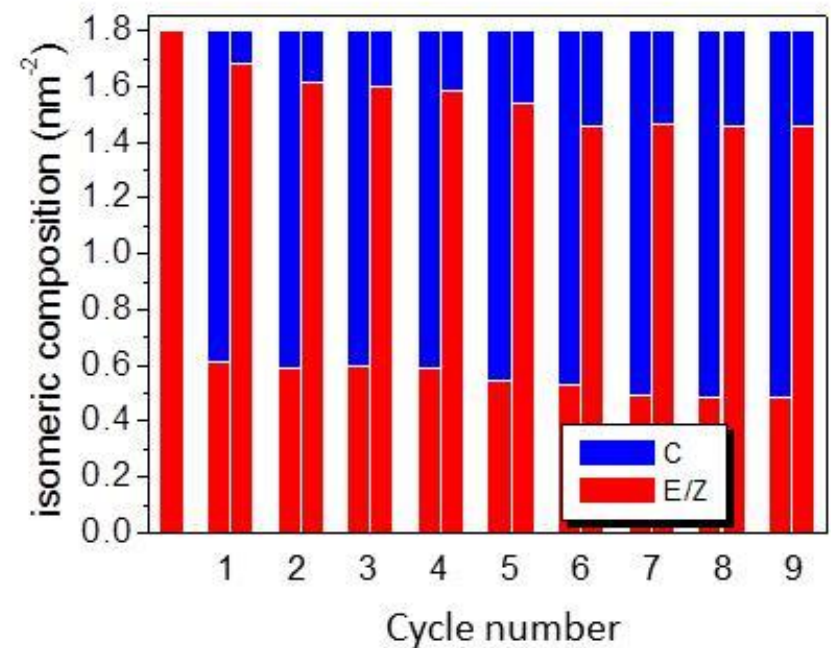

Figure 2:

(a) Time dependence of $\alpha$ (left scale) and surface density of $E / Z$ isomers (right scale) during successive UV and Vis partial photoconversion of a nominal pure E monolayer. The initial surface is an E-surface (1.8 E-isomers $\left./ \mathrm{nm}^{2}\right)$. (b) Isomeric composition of the ML at PSSs: for each UV/Vis illumination cycle a pair of bars gives the extrapolated isomeric composition at $\mathrm{PSS}_{\mathrm{UV}}$ (left bar) and PSS $\mathrm{Vis}_{\text {is }}$ (right bar) of individual switching cycles. The single bar on the left corresponds to the as prepared E-terminated surface. 


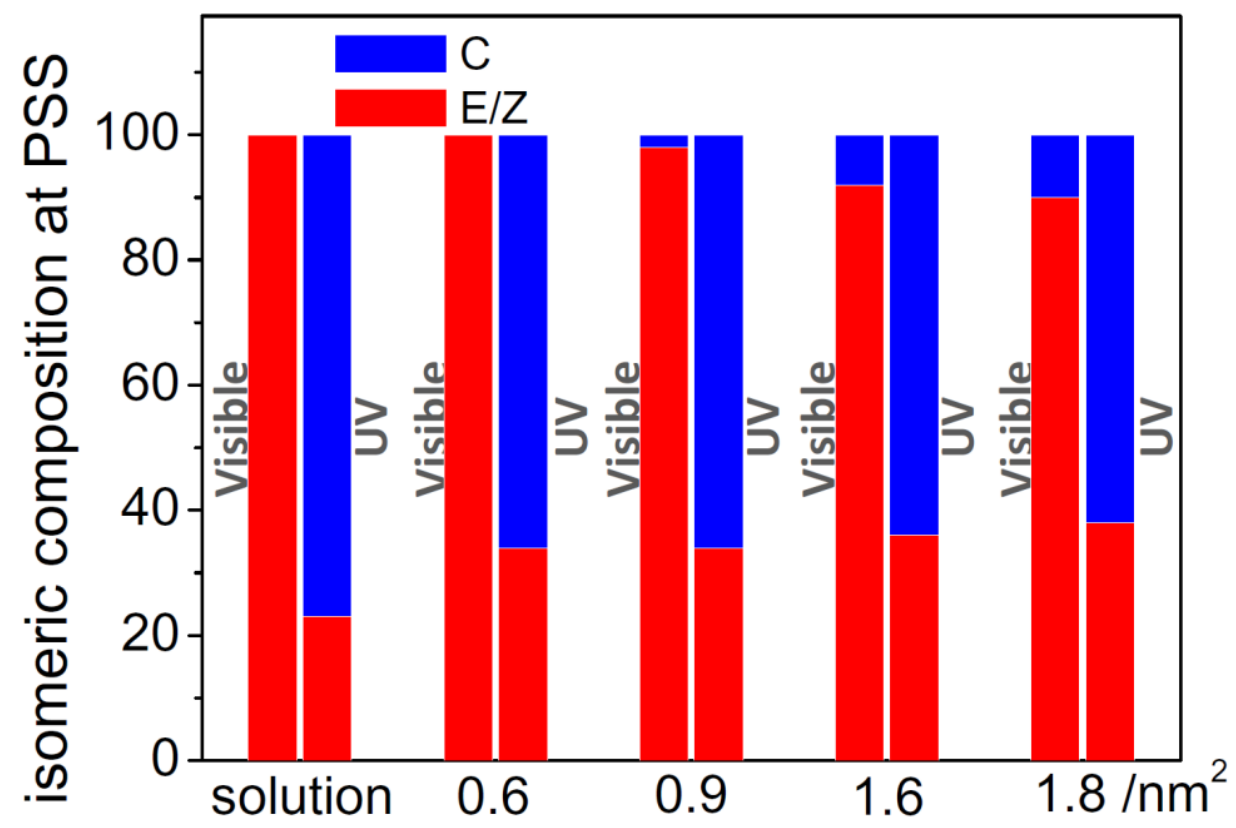

\section{Figure 3:}

Isomeric composition at $\mathrm{PSS}_{\mathrm{Vis}}$ and $\mathrm{PSS}_{\mathrm{UV}}$ in solution and at surfaces, starting with a pure Csolution $(20 \mu \mathrm{mol} / \mathrm{L})$ or $\mathrm{C}$-monolayer with various densities of photochromic molecules. In each case a pair of bars gives PSS $_{\mathrm{Vis}}$ (left bar) and $\mathrm{PSS}_{\mathrm{UV}}$ (right bar). 

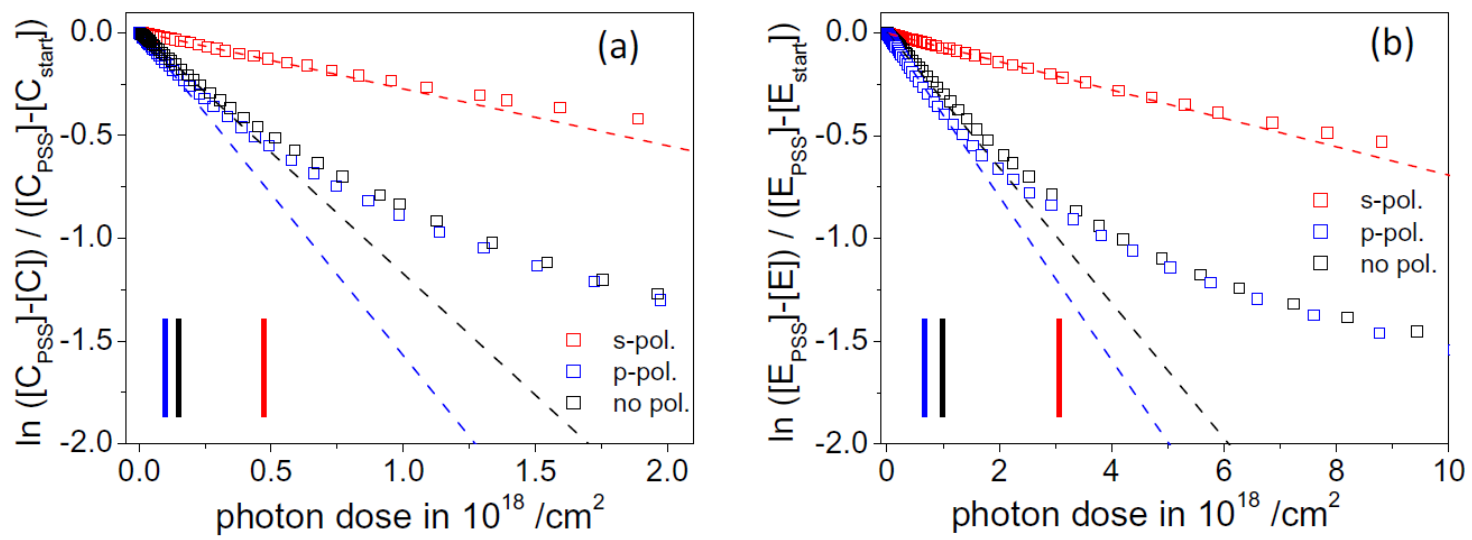

\section{Figure 4:}

Influence of light polarization on the photoswitching. (a-b) Progression of the reaction during $\mathrm{UV}$ (left) and Vis (right) illumination. The surface density of fulgimides $(\mathrm{C}+\mathrm{E} / \mathrm{Z})$ is $1.6 \mathrm{~nm}^{2}$. In (a) the starting surface is composed of $1.1 \mathrm{E}$-isomers $/ \mathrm{nm}^{2}$ and $0.5 \mathrm{C}$-isomers $/ \mathrm{nm}^{2}$. In (b) the starting surface contains 0.6 E-isomers $/ \mathrm{nm}^{2}$ and $1.0 \mathrm{C}$-isomers $/ \mathrm{nm}^{2}$ for the UV (resp. visible) illumination. Symbols correspond to experimental data, and dashed lines to linear fits. Vertical bars mark for each plot the maximum photon dose $p^{*}$ up to which the cross section remains constant. 


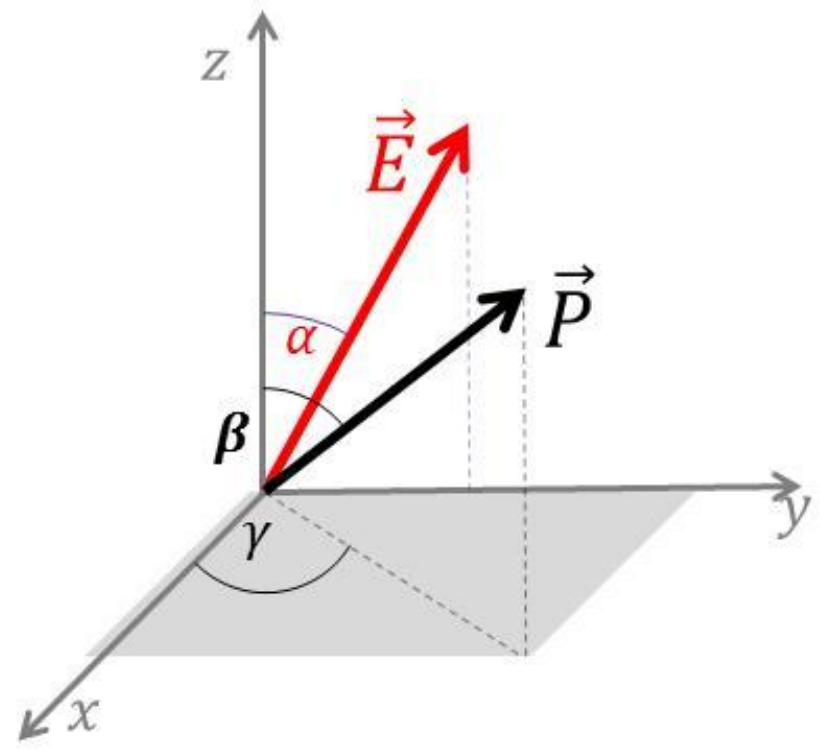

\section{Figure 5:}

Definition of angles used to calculate $J$ in case the photochromic molecules are constrained in a defined direction of space (see Eq. 2 and text). 
(a)

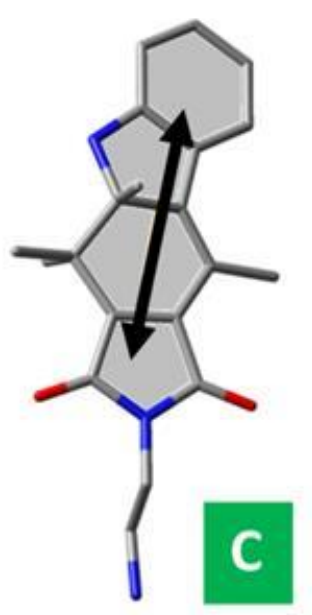

relative

energies: $\quad 13.5 \mathrm{~kJ} \mathrm{~mol}^{-1}$ (b)

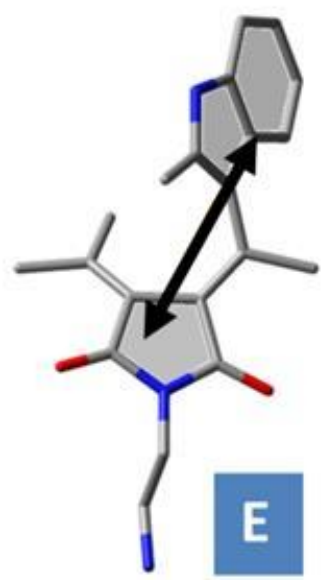

$0 \mathrm{~kJ} \mathrm{~mol}^{-1}$ (c)

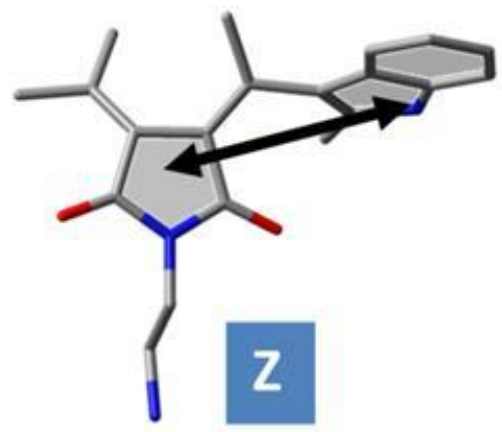

$1.9 \mathrm{~kJ} \mathrm{~mol}^{-1}$

\section{Figure 6:}

Minimized geometries and relative energies of the isolated amino-fulgimide in its C (a), E (b) and $\mathrm{Z}$ (c) forms as calculated by DFT (B3LYP functional, 6-3111+G(d,p) basis set, in vacuum), with the indication of the direction of the electronic transition dipole moment for each isomer (black double arrows) computed by TD-DFT. 
(a)

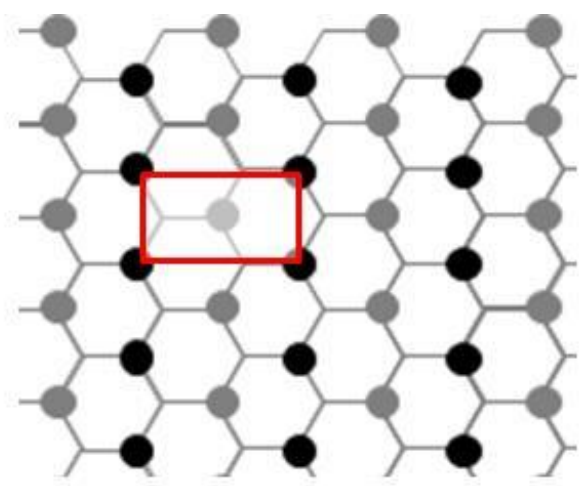

(b)

top view

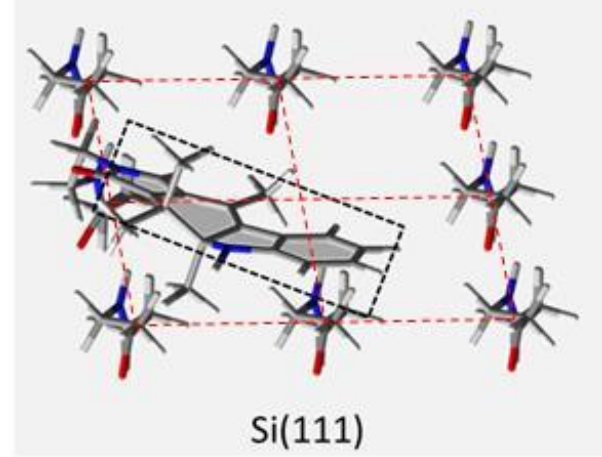

(c)

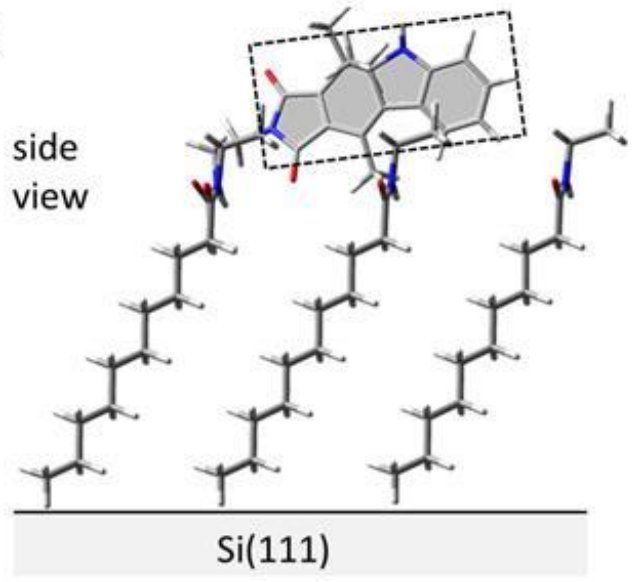

\section{Figure 7:}

(a) Atomic model of a perfectly grafted $\mathrm{Si}(111)$ surface where grey circles are $\mathrm{H}$ bonds and black circles feature the anchoring sites of the alkyl monolayer according to a ( $3.84 \AA$ x 6.65 $\AA$ ) unit cell (red rectangle). (b) and (c) top and side views of a 3 x 3 cell with a vacancy at the centre and a $\mathrm{C}$-fulgimide immobilized with an orientation consistent with the experimental results $\left(\beta=74^{\circ}\right)$; The top view is tilted with respect to the surface normal which explains that the red mesh is not rectangular. The side view shows that the fulgimide group is partly embedded in the molecular layer. This configuration is made possible by the presence of a vacancy in the centre of the red mesh. A similar configuration is found for the E form. See text for details. 